\title{
Vitamin D Serum Level in Patients with Schizophrenia in West Java, Indonesia
}

\author{
Nofan Pratama Maulana, ${ }^{1}$ Tuti Kurnianingsih, ${ }^{2}$ Sumartini Dewi ${ }^{3}$ \\ ${ }^{1}$ Faculty of Medicine Universitas Padjadjaran, Indonesia, ${ }^{2}$ Department of Phsyciatry \\ Faculty of Medicine Universitas Padjadjaran/Dr. Hasan Sadikin General Hospital Bandung, \\ Indonesia, ${ }^{2}$ Department of Internal Medicine Faculty of Medicine Universitas Padjadjaran/ \\ Dr. Hasan Sadikin General Hospital Bandung, Indonesia
}

\section{Abstract}

Background: Vitamin D is an immune-regulator that influences the neuro-inflammation process in schizophrenia. The study aimed to explore the vitamin D serum level in schizophrenic patients at the outpatient clinic of the Provincial Mental Hospital, West Java, Indonesia.

Methods: A quantitative descriptive study was conducted in November-December 2019 using secondary data of all-male schizophrenic patients at the Provincial Mental Hospital. Data collected were demographic characteristics, clinical characteristics, and vitamin D Level. Scoring was then performed using Positive and Negative Syndrome Scale (PANSS) and Montreal Cognitive Assessment (MoCA) to assess the positive and negative symptoms as well as the cognitive symptoms based on the serum vitamin D levels.

Results: All male schizophrenic patients had a low serum vitamin D level (mean16.67 $\pm 5.6 \mathrm{ng} / \mathrm{ml}$ ) with $64.52 \%$ of them had vitamin D deficiency $(<20 \mathrm{ng} / \mathrm{ml})$. Interestingly, patients with vitamin D deficiency had a negative symptom (mean22.1 $\pm 5.6 \mathrm{ng} / \mathrm{ml}$ ) whereas those with vitamin D insufficiency (20-29 ng/ $\mathrm{ml}$ ) had a positive symptom (mean $18.55 \pm 5.6 \mathrm{ng} / \mathrm{ml}$ ). Furthermore, patients with vitamin D deficiency had a lower impaired cognitive function value (mean $19.7 \pm 3.4 \mathrm{ng} / \mathrm{ml}$ ) compared to those with vitamin D insufficiency.

Conclusions: A low vitamin D serum level in schizophrenic patients may play a role in the pathogenesis of this disorder. Further studies are needed to confirm this finding.

Keywords: Montreal cognitive assessment, positive and negative Syndrome scale, schizophrenia, vitamin D

\section{Introduction}

Schizophrenia is a psychiatric disorder that affects thought processes and causes mental and social function disorders. Characteristics of schizophrenia symptoms are divided into three groups, including positive symptoms such as delusions and hallucinations; negative symptoms such as blunted affect and apathetic; and cognitive symptoms such as attention and memory disorders. ${ }^{1}$ The World Health Organization (WHO) data shows the range of schizophrenic patients in the world is 21 million people, ${ }^{2}$ and the Basic Health Research (Riset Kesehatan Dasar, Riskesdas) data shows the proportion of households with schizophrenia or psychosis in Indonesia is about seven per 1000 population. ${ }^{3}$ The etiology of schizophrenia is still unknown. The current hypothesis explains that the manifestations of schizophrenia occur due to abnormalities or pathophysiological changes in the transmission of neurotransmitters, such as dopamine and glutamate, in the central nervous system. Mesolimbic and mesocortical dopaminergic pathways are responsible for manifesting positive symptoms, negative symptoms, and cognitive symptoms. The rate of glutamate release can affect the dopaminergic pathways through the cortico-brainstem glutamate pathways. Increased glutamate release influences the rate of dopamine release which can trigger the clinical symptoms of schizophrenia. ${ }^{4}$ Glutamate transmission can

Correspondence: Nofan Pratama Maulana, Faculty of Medicine, Universitas Padjadjaran/Dr. Hasan Sadikin General Hospital, Jalan Raya Bandung-Sumedang Km. 21, Jatinangor, Sumedang, Indonesia, Email: nofanpratama@gmail.com 
be influenced by several things, one of which is NMDA receptor hypofunction caused by the neuroinflammation process. ${ }^{5}$ Vitamin D which functions as an immune-regulator is thought influence the neuroinflammation process in schizophrenia. ${ }^{6}$ Risk factors for vitamin D deficiency such as geographic location, BMI, age, and prenatal infections were also found to be related to the risk of developing schizophrenia.?

Vitamin D is a fat-soluble steroid hormone obtained from the synthesis of pro-vitamin D in the skin with the help of exposure to ultraviolet light and food intake. Vitamin D has two forms according to the source, namely vitamin D3 and vitamin D2. Both forms must undergo two hydroxylation processes to become active form $1.25(\mathrm{OH}) 2 \mathrm{D}^{8}$ Besides having a role in bone metabolism and calcium homeostasis, vitamin $\mathrm{D}$ also plays a role in the function and development of the brain and immune system. Researchers have found that the central nervous system can synthesize and metabolize vitamin D. Vitamin D receptors and enzymes that play a role in vitamin D metabolism are found scattered in various regions of the brain such as the hippocampus, cortex, and limbic system. ${ }^{9}$ There are various psychiatric disorders, such as Alzheimer's, mood disorders, major depression, and psychosis or schizophrenia reported to have involvement with vitamin D. ${ }^{10}$

Research conducted in Europe in schizophrenia patients with the first psychosis episode showed that all patients had lower vitamin D levels than controls. ${ }^{11}$ Another study in Medan, North Sumatra found a lower mean serum vitamin D level than normal levels among schizophrenia patients from the Batak tribe. ${ }^{12}$ Various studies have found a decrease in vitamin D levels in schizophrenic patients, but no similar studies yet in West Java. Therefore, this study aimed to explore the vitamin D levels in schizophrenia patients in West Java Province.

\section{Methods}

This was quantitative descriptive research conducted in November-December 2019. In total, 31 schizophrenic patients data who met the inclusion criteria were collected, such as age 18-45 years, male, used atypical antipsychotic drugs, could speak Indonesian, and both patients and families signed a written consent letter to participate in the

Table 1 Demographic Characteristic of Subjects

\begin{tabular}{lc}
\hline \multicolumn{1}{c}{ Characteristics } & $\begin{array}{c}\text { Subjects } \mathbf{( n = 3 1 )} \\
\mathbf{n}(\mathbf{\%})\end{array}$ \\
\hline Age (years) & $35.23 \pm 6.8$ \\
Mean \pm SD & 36 \\
Median & $24-45$ \\
Range & \\
Education & $6(19.35)$ \\
Elementary School & $3(9.68)$ \\
Junior High School & $20(64.52)$ \\
Senior High School/Vocational High School & $2(6.45)$ \\
College & \\
Marital Status & $22(71.97)$ \\
Not Married & $8(25.8)$ \\
Married & $1(3.22)$ \\
Divorced & \\
Occupation & $19(61.29)$ \\
Working & $12(38.71)$ \\
Unemployed &
\end{tabular}

Notes: $\mathrm{n}=$ frequency, $\mathrm{SD}=$ Standard Deviation 
Table 2 Clinical Characteristics of Subjects

\begin{tabular}{|c|c|}
\hline Characteristics & $\begin{array}{c}\text { Subjects }(n=31) \\
n(\%)\end{array}$ \\
\hline \multicolumn{2}{|l|}{ Age of Onset (years) } \\
\hline Mean \pm SD & $24.87 \pm 6.5$ \\
\hline Median & 23 \\
\hline Range & $8-41$ \\
\hline \multicolumn{2}{|l|}{ Body Mass Index $\left(\mathrm{kg} / \mathrm{m}^{2}\right)$} \\
\hline Mean \pm SD & $22.56 \pm 3.3$ \\
\hline$<18.5$ (Underweight) & $3(9.68)$ \\
\hline 18.5-24.9 (Normal) & $23(74.19)$ \\
\hline 25.5-29.9 (Overweight) & $4(12.9)$ \\
\hline$\geq 30.0$ (Obese) & $1(3.22)$ \\
\hline \multicolumn{2}{|l|}{ Antipsychotics Used } \\
\hline Clozapine, Risperidone, Trihexyphenidyl & $22(70.94)$ \\
\hline Risperidone, Trihexyphenidyl & $9(29.03)$ \\
\hline \multicolumn{2}{|l|}{ Serum Vitamin D Levels (ng/ml) } \\
\hline Mean \pm SD & $16.67 \pm 5.6$ \\
\hline Median & 16.76 \\
\hline Range & $8.02-27.75$ \\
\hline \multicolumn{2}{|l|}{ PANSS Positive Symptoms } \\
\hline Mean \pm SD & $17 \pm 6$ \\
\hline \multicolumn{2}{|l|}{ PANSS Negative Symptoms } \\
\hline Mean \pm SD & $21 \pm 6$ \\
\hline \multicolumn{2}{|l|}{ MoCACognitive Symptoms } \\
\hline Mean \pm SD & $20 \pm 4$ \\
\hline
\end{tabular}

study. The exclusion criteria were intellectual disability, anxiety and uncooperative, and the use of psychotropic drugs other than a typical antipshychotics. The study protocol was granted by the Research Ethics Committee Universitas Padjadjaran, number 1350/UN6. $\mathrm{KEP} / \mathrm{EC} / 2019$.

The vitamin D 25(OH)D serum levels were measured using an electro chemiluminescence immunoassay analyzer (ECLIA) and classified into normal (>30 ng/ml), insufficiency (20-29 $\mathrm{ng} / \mathrm{ml})$, and the deficiency $(<20 \mathrm{ng} / \mathrm{ml})$. The assessment of positive and negative symptoms was carried out based on the Positive and Negative Syndrome Scale (PANSS) to assess the severity of seven domains of each symptom. A value of one indicated no symptoms and a value of seven indicated extreme symptoms. Domain with a value of three or greater indicated symptom manifestations. Cognitive function disorders were classified based on the total score from the Montreal Cognitive Assessment (MoCA) with a score of $>26$ indicated no cognitive impairment, and a score of $<26$ indicated a cognitive impairment.

The scores then adjusted to the patient's educational history by adding a value of one to patients with an education of less than four years. The data was presented in the form of tables that were valued for frequency and percentage (IBM SPSS Statistics software).

\section{Results}

Of 31 schizophrenic patients, the mean age was 35.23 (SD + 6.82; range $24-45$ years). The last educational history was predominantly high school or equivalent (64.52\%), already had a 
Table 3 Serum Vitamin D Levels

\begin{tabular}{lc}
\hline \multicolumn{1}{c}{ Serum Vitamin D Levels(ng/ml) } & $\begin{array}{c}\text { Subjects (n= 31) } \\
\mathbf{n}(\mathbf{\%})\end{array}$ \\
\hline Insufficiency & $11(35.48)$ \\
Deficiency & $20(64.52)$ \\
\hline
\end{tabular}

Table 4 PANSS and MoCA Scoring based on Serum Vitamin D Levels

\begin{tabular}{|c|c|c|}
\hline \multirow{2}{*}{ PANSS and MoCA Scoring } & \multicolumn{2}{|c|}{ Serum Vitamin D Levels } \\
\hline & Insufficiency $(\mathrm{n}=11)$ & Deficiency $(n=20)$ \\
\hline \multicolumn{3}{|l|}{ PANSS } \\
\hline Positive Symptoms & $18.55 \pm 5.6$ & $15.95 \pm 6.6$ \\
\hline Negative Symptoms & $19.55 \pm 5.7$ & $22.1 \pm 5.6$ \\
\hline \multicolumn{3}{|l|}{ MoCA } \\
\hline Cognitive Symptoms & $20.45 \pm 3.9$ & $19.7 \pm 3.4$ \\
\hline
\end{tabular}

job (61.29\%), but not married yet (71.92\%) (Table 1).

The age of onset of the subjects was found to be quite young (mean $24.87+6.5$ years). Most subjects had a normal body mass index $(74.19 \%)$ and used clozapine, risperidone, and trihexyphenidyl (70.94\%) as the antipsychotics. Mean serum vitamin D levels showed decreased levels compared to normal $(16.67 \pm 5.6)$. The average value of PANSS and MoCA showed that almost all subjects had a mean positive symptoms value of $17 \pm 6$, mean negative symptoms value of $21 \pm 6$, and mean cognitive symptoms of $20 \pm 4$ (Table 2).

Serum vitamin D levels of all of the subjects were found to be lower than normal levels and most had vitamin D deficiency (64.52\%) as depicted in Table 3 . In Table 4, the subjects with vitamin $D$ deficiency hade higher negative symptom (22.1 \pm 5.6$)$ and lower cognitive impairment values $(19.7 \pm 3.4)$, while subjects with vitamin D insufficiency had a higher positive symptom value $(18.55 \pm 5.6)$.

\section{Discussions}

In this study, all schizophrenia patients have low serum vitamin D levels, similar to research conducted in the United Kingdom, showing that schizophrenia patients have a low serum vitamin D level. ${ }^{11,13}$ This result is also similar in other parts of Indonesia, in Medan and Surabaya. 12,14 The decrease of serum vitamin D levels in the study might be caused by several factors such as lack of sun exposure, lack of intake of foods containing vitamin D, the presence of genetic factors that affect vitamin D metabolism, and possible drug interactions. ${ }^{15}$

The findings regarding low vitamin $D$ levels support the hypothesis of the role of vitamin D in the pathogenesis of schizophrenia. Vitamin $\mathrm{D}$ has a function in the immune system and inflammation as a regulator of activation, proliferation, and differentiation of immune and inflammatory cells. This function enables vitamin $\mathrm{D}$ to reduce the production of type 1 pro-inflammatory cytokines and increase the production of type 2 anti-inflammatory cytokines. In vitamin D deficiency, regulation of the immune and inflammatory systems mediated by vitamin $\mathrm{D}$ is disrupted, causing an imbalance in inflammatory cytokine levels and increasing pro-inflammatory cytokines. ${ }^{6}$

Type 2 immune responses initiated by inflammatory cytokines can inhibit indoleamine 2,3-dioxygenase (IDO) enzyme from the metabolism of tryptophan/kynurenine. The IDO enzyme functions as a regulator of tryptophan/ kynurenine metabolism by converting tryptophan to kynurenine, then kynurenine can produce neuroactive metabolites of kynurenic acid with the help of the enzyme kynurenine aminotransferase. Kynurenic acid is a natural antagonist for N-methyl-D-Aspartate (NMDA) receptors. The NMDA receptor is one of the ionotropic receptors used by glutamate. Increased NMDA receptor antagonisms cause hypofunction of the receptor, leading 
to increased glutamate release. The excess release of glutamate can affect the activity of the dopaminergic pathway and the release of dopamine. $^{5}$

Physiologically, dopamine works through several dopaminergic pathways. Pathways that play a role in the pathogenesis of schizophrenia are mesolimbic and mesocortical pathways. In the mesolimbic pathway, dopamine is sent from the ventral tegmental area to the nucleus accumbens, which functions in the regulation of emotional behavior. In the mesocortical pathway, dopamine sent from the ventral tegmental area to the prefrontal cortex, especially in the dorsolateral region. High glutamate release in the ventral tegmental area results in the increased mesolimbic pathway and decreased mesocortical pathway activity. Increased mesolimbic activity is thought to cause positive symptoms such as delusions and hallucinations, whereas decreased dopamine release in the mesocortical pathway is thought to cause negative symptoms and cognitive symptoms. ${ }^{4}$

In this study, the PANSS value of negative symptoms was higher in patients with vitamin D deficiency. This is in accordance with a study in Turkey that found patients with vitamin D deficiency had higher negative symptom values than patients with vitamin D insufficiency. ${ }^{7}$ This study also found subjects with vitamin D deficiency experience impaired cognitive function with a low MoCA value. This finding is in accordance with a study in Norway which found schizophrenia patients with vitamin D deficiency had cognitive impairment. ${ }^{16}$ Excessive reduction of serum vitamin D levels in vitamin D deficiency increased the release of excess glutamate and dopamine. These conditions further reduce the release of dopamine into the prefrontal cortex, thereby increasing the severity of negative and cognitive symptoms in patients.

The PANSS value of positive symptoms in this study was found to be higher in patients with vitamin D insufficiency. However, our result is not in accordance with research conducted in Turkey ${ }^{7}$ which found that the value of positive symptoms (SAPS) was higher in patients with vitamin D deficiency. Our study is an outpatient schizophrenic patient who has received atypical antipsychotic therapy so that the patient has experienced a decrease in positive symptoms. A typical antipsychotic therapy has dopamine receptor antagonistic properties so it works by reducing the level of dopamine release in the brain. The effects of antipsychotic therapy are more effective in dealing with positive symptoms than negative and cognitive symptoms. Antipsychotic therapy can also affect the body's metabolism, but no findings of the interactions between antipsychotic drugs and serum vitamin D levels.

The limitation of this study is that our patients are outpatients who have received atypical antipsychotic therapy and some of them get additional clozapine therapy. Further research is needed with data that are more complete to eliminate confounding factors for serum vitamin D levels.

To conclude, all schizophrenic patients have low serum vitamin D levels. Schizophrenic patients with vitamin D deficiency have higher negative symptom values and lower cognitive impairment values, whereas schizophrenia patients with vitamin D insufficiency have higher positive symptom values.

\section{References}

1. Patel KR, Cherian J, Gohil K, Atkinson D. Schizophrenia: overview and treatment options. P T. 2014;39(9):638-45.

2. World Health Organization (WHO). Fact Sheets: Schizophrenia. Geneva: World Health Organization; 2018.

3. Kementrian Kesehatan Republik Indonesia. Penyakit tidak menular dan kesehatan jiwa. Hasil Utama Riskesdas. Jakarta: Badan Penelitian dan Pengembangan Kesehatan; 2018. p.78-80.

4. Stahl SM. Stahl's essential psychopharmacology: neuroscientific basis and practical applications. 4th ed. New York, UK: Cambridge University Press; 2013. p. 79-128.

5. Müller N. Inflammation in schizophrenia: pathogenetic aspects and therapeutic considerations. Schizophr Bull. 2018;44(5):973-82.

6. Colotta F, Jansson B, Bonelli F. Modulation of inflammatory and immune responses by vitamin D. J Autoimmun. 2017;85:78-97.

7. BulutSD, BulutS,Atalan DG, BerkolT, Gürçay $\mathrm{E}$, Türker T, et al. The relationship between symptom severity and low Vitamin D levels in patients with schizophrenia. PLoS One. 2016;11(10):e0165284.

8. Nair R, Maseeh A. Vitamin D: the "sunshine" vitamin. J Pharmacol Pharmacother. 2012;3(2):118-26.

9. Cui X, Gooch H, Petty A, McGrath JJ, Eyles D. Vitamin D and the brain: Genomic and non-genomic actions. Mol Cell Endocrinol. 2017;453:131-43. 
10. Graham KA, Keefe RS, Lieberman JA, Calikoglu AS, Lansing KM, Perkins DO. Relationship of low vitamin D status with positive, negative and cognitive symptom domains in people with first-episode schizophrenia. Early Interv Psychiatry. 2015;9(5):397-405.

11. Salavert J, Grados D, Ramiro N, Carrión MI, Fadeuilhe C, Palma F, et al. Association between Vitamin D status and schizophrenia: a first psychotic episode study. J Nerv Ment Dis. 2017;205(5):40912.

12. Prasanty N, Amin MM, Effendy E, Simbolon J. Low Vitamin D serum level increases severity symptoms in schizophrenic patients measured by Positive and Negative Symptoms Scale (PANSS) in Batak Tribe Sumatera Utara, Medan-Indonesia. Bali Med J. 2018;7(1):249-54.

13. Crews M, Lally J, Gardner-Sood P, Howes
O, Bonaccorso S, Smith S, et al. Vitamin D deficiency in first episode psychosis: a case-control study. Schizophr Res. 2013;150(2-3):533-7.

14. Simangunsong AD, Karimah A, Marpaung FR. Correlation between serum vitamin $\mathrm{d}$ levels and severity of schizophrenia patients in the Dr. Soetomo Hospital. Indonesian Journal of Clinical Pathology and Medical Laboratory. 2019;25(3):2506.

15. Akinlade KS, Olaniyan OA, Lasebikan VO, Rahamon SK. Vitamin D levels in different severity groups of schizophrenia. Front Psychiatry. 2017;8:105.

16. Nerhus M, Berg AO, Simonsen C, Haram M, Haatveit B, Dahl SR, et al. Vitamin D deficiency associated with cognitive functioning in psychotic disorders. J Clin Psychiatry. 2017;78(7):e750-7. 